\title{
Adaptive noise cancellation system for low frequency transmission of sound in open fan aircraft
}

\author{
Steven Griffin*, Adam Weston and Jeff Anderson \\ Boeing Company, Seattle, WA, USA \\ Received 9 August 2012 \\ Revised 15 March 2013 \\ Accepted 25 March 2013
}

\begin{abstract}
This paper describes the use of a structural/acoustic model of a section of a large aircraft to help define the sensor/actuator architecture that was used in a hardware demonstration of adaptive noise cancellation. Disturbances considered were representative of propeller-induced disturbances from an open fan aircraft. Controller on and controller off results from a hardware demonstration on a portion of a large aircraft are also included. The use of the model has facilitated the development of a new testing technique, closely related to modal testing, that can be used to find good structural actuator locations for adaptive noise cancellation.
\end{abstract}

Keywords: Active noise control, open fan aircraft

\section{Introduction}

A wealth of technical research has accumulated starting in the late 1980's exploring the concept of active acoustic control in aircraft interiors. Much of this work shows a direct correlation with fuel prices as more fuel-efficient aircraft such as turboprop and open fan power plants also tend to create relatively high amplitude propeller-induced disturbance. Because much of the annoying cabin noise is caused by propellers and is tonal and at a relatively low frequency, reducing the sound with adaptive noise cancellation becomes much more tractable than the more general problem of mostly broadband, flow-induced noise (either turbulent boundary layer or jet noise).

In one of the first aircraft implementations of adaptive noise cancellation (ANC) [8], a flight test on a B.Ae. 748 aircraft showed reductions at the fundamental mode and first two harmonics of the propeller using interior microphones and loudspeakers. It was also demonstrated that the filtered x LMS (FxLMS) algorithm performed well during engine run up on the ground, where the system levels at each microphone varied widely with time, representing a less stationary system than in flight. In the same time frame, another flight demonstration of ANC [6] on the B.Ae. 748 showed reductions of amplitude at the same frequencies and demonstrated similar results between actual measured performance and predictions of performance based on transfer function measurements and noise. Boeing also successfully flight tested ANC on the DeHaviland Dash 8, although the results were not published. The first demonstration of active structure-borne noise reduction on a full-size aircraft [23] used the aft section of a Douglas DC-9 to show that a small number of structural actuators provide good global control when the error sensors are microphones and can also reduce vibration when the error sensors are accelerometers. These early flight

*Corresponding author: Steven Griffin, Boeing Company, Seattle, WA, USA. E-mail: steven.f.griffin@ boeing.com. 


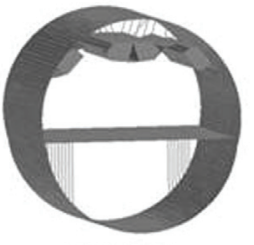

structure

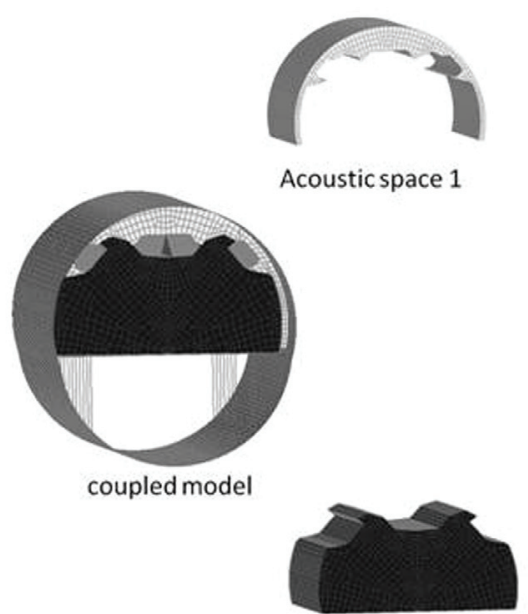

Acoustic space 2

Fig. 1. Structural and acoustic portions of the model.

and ground tests paved the way for at least two commercially available turboprop aircraft from Saab (340B Plus) and Bombardier (Dash 8 Q Series) with active noise and vibration control systems available as an option or standard equipment. The DC-9 has tuned absorbers as OEM equipment to address local vibration related to engine tones. More recently, an actively tuned version was offered by Barry Controls that detects the engine vibration frequency and continuously tunes the absorber [1].

In general, most work that has been published on finding preferred or optimal locations for sensors and actuators is based on analysis [16,24,25] or demonstration on relatively simple laboratory testbeds [14,17]. None of the work to date has incorporated a structural/acoustic model of a section of a large aircraft to help define the sensor/actuator architecture in a hardware demonstration. The use of such a model in this work has also made possible the development of a new testing technique, closely related to modal testing, that can be used to find good actuator locations for adaptive noise cancellation.

\subsection{Analytical model}

The analytical model started with a coupled structural/acoustic finite element model. Figure 1 illustrates the structural and acoustic portions of the model. The structural portion of the model includes a large portion of a Boeing 767-200, known as a barrel, with dimensions $6.1 \mathrm{~m}$ long and $4.9 \mathrm{~m}$ diameter. The skin of the barrel and the interior facing structure are included in the model. The acoustic space is broken up into two, noncommunicating parts: the interior acoustic space designated as acoustic space 2 and the acoustic space between the exterior skin and the interior structure designated as acoustic space 1. The structural portion of the model includes 19639 bar, rod and laminated plate elements and 13574 nodes. Acoustic space 1 includes 8064 solid elements and 11154 nodes, and acoustic space 2 includes 23168 solid elements and 25344 nodes.

The structural/acoustic analytical model was assembled using the modal interaction approach [10]. The motivation for using this approach was the resulting state space model format in Matlab, where it was possible to develop different control strategies to be used in the hardware implementation. This approach has been shown to give similar answers to the coupled structural/acoustic analysis capability in NASTRAN using the modal method for analyses that did not include active control.

The questions to be answered using the analytical model were

- Locations of error sensors?

The choices were between two regions, one located closer to the ears of the passengers and one located closer to the disturbance input. 


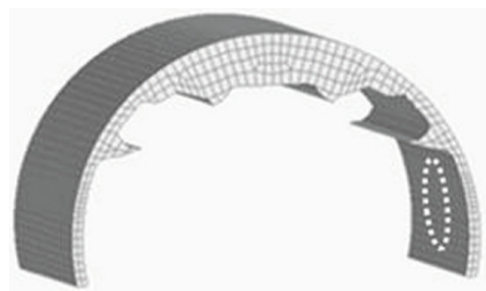

Acoustic space 1 with region 1 shown with dashed lines. Region extends through thickness of space

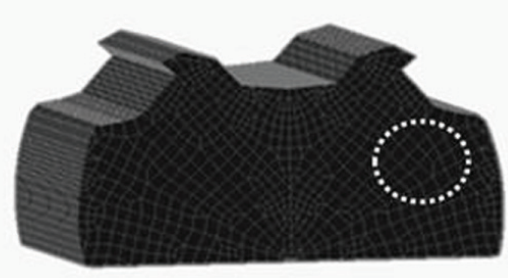

Acoustic space 2 with region 2 shown with dashed lines. Region extends over length of space

Fig. 2. Definitions of regions 1 and 2.

- Locations of actuators?

In this case, there were many more choices, including all the possible locations on the skin that were on the same side of the barrel as the disturbance.

- Force capabilities and weight of actuator?

This question was related to the assumed amplitude of the uncontrolled signals at the error sensors. This was important in selecting an actuator for the experimental portion of the work.

The disturbance model used was assumed to be an idealized localized source. All the normal components of skin nodes on one side of the airplane within a 50 inch diameter circle were forced to move in phase to represent a low frequency plane wave. The circle started slightly above the representation of the floor attachment and extended over approximately the height of one seated passenger. A more accurate representation of the source was not attempted due to the uncertainty in source disturbance models.

\section{Sensor locations}

Two separate sensor locations were analyzed based on proximity to the disturbance input, region 1, and proximity to the location where sound was to be minimized, region 2 . This region extends over the length of the interior acoustic space and it encompasses the region where the heads of the passengers are located when seated. The locations in region 1 have the advantage of allowing the sensors to be placed out of passenger contact between the skin and trim panels but have the disadvantage of not guaranteeing noise reduction at passenger locations. The number of error sensors considered were 16, 12 and 8. Regions 1 and 2 are shown in Fig. 2.

\section{Actuator location metric}

Actuator locations were selected based on the assumption that a good location was one that created a field at all sensor locations that was similar to that created by the disturbance both in amplitude and phase at a given frequency. Using the structural/acoustic model and the idealization of the disturbance, it was possible to compute a transfer function vector between the disturbance and all the error sensor locations. At a given frequency, the value of the transfer function disturbance vector, $\Phi$, is a complex quantity that gives amplitude and phase relationships between the disturbance and the error sensors. An actuator vector, $\Psi$, can be calculated for a candidate actuator location by using the model to generate the transfer function vector between an out-of-plane force input at a given location and the resulting response at the error sensors. It is assumed, in this calculation, that the added mass and dynamics of the actuator that would impart this force in a control system does not significantly modify this response. The complex vector quantity from the disturbance and from the candidate actuator location can then be compared in much the same way that complex mode shapes are compared for similarity. The two approaches used for this comparison were the Modal Assurance Criteria (MAC) [2] and a similar approach named the vector normalization approach. 


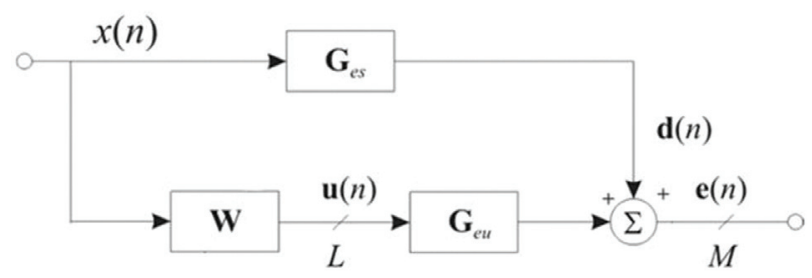

Fig. 3. Block diagram of feedforward control problem with $\boldsymbol{L}$ actuators and $\boldsymbol{M}$ sensors.

The MAC is defined as

$$
M A C=\frac{\left|\Phi^{T} \psi^{*}\right|^{2}}{\left(\Phi^{T} \Phi^{*}\right)\left(\psi^{T} \psi^{*}\right)}
$$

The resulting scalar quantity is 1 if the vectors are consistent (linearly related) and 0 if the vectors are not consistent.

The vector normalization approach is formed by first normalizing the disturbance vector, $\Phi$, by the component with the largest absolute amplitude creating $\Phi^{\prime}$. Physically, this represents the microphone location with the highest response due to the disturbance at a given frequency. The actuator vector is then normalized by the component at the same location creating $\Psi^{\prime}$. The two complex vectors can then be subtracted and their similarity is indicated by the inverse of the sum of the difference as

$$
\text { vecnorm }=\frac{1}{\sqrt{\left(\Phi^{\prime}-\psi^{\prime}\right)^{T}\left(\Phi^{\prime}-\psi^{\prime}\right)}}
$$

The larger the vecnorm scalar quantity is, the more similar the disturbance and actuator vectors are, with the quantity approaching infinity for identical vectors and 0 for vectors that are very dissimilar. Both the MAC and the vecnorm were calculated to determine which actuator locations ranked highest in their ability to create a field that was similar to the disturbance.

To evaluate the highest ranked actuator locations in their ability to quiet the disturbance, an additional performance metric was introduced. Assuming a block diagram of the feedforward control problem shown in Fig. 3 where $x(n)$ is the disturbance reference signal, the error signals can be defined as

$$
\mathbf{e}(n)=\mathbf{d}(n)+\mathbf{G}_{\boldsymbol{e u}} \mathbf{u}(n)
$$

at a given frequency $n=e_{s}^{j \omega T}$ where $\mathbf{G}_{\boldsymbol{e u}}$ is the secondary path transfer function matrix, $\mathbf{u}(n)$ is the vector of control signals and $\mathbf{d}(n)$ is the vector of disturbance signals to be canceled. The vector of optimal control signals for cancellation [7] at the error sensors is

$$
\mathbf{u}_{\mathbf{o}}=-\mathbf{G}_{e u}^{+} \mathbf{d}
$$

where $(\cdot)+$ denotes the pseudo-inverse of the term inside parentheses. After substituting

$$
\mathbf{d}=\mathbf{G}_{e s} x(n)
$$

where $\mathbf{G}_{e s}$ is the primary transfer function matrix and $x(n)$ is the disturbance input force, the cancellation metric defined as

$$
\frac{-\mathbf{G}_{\boldsymbol{e u}} \mathbf{G}_{\boldsymbol{e u}}^{+} \mathbf{G}_{\boldsymbol{e s}} x(n)+\mathbf{G}_{\boldsymbol{e s}} x(n)}{\mathbf{G}_{\boldsymbol{e s}} x(n)}
$$

gives the resulting sound level at sensor locations in the region to be quieted after optimal cancellation normalized by the sound level with no control applied. 
Table 1

Means of performance metrics for configurations 1 and 2 using optimal compensator

\begin{tabular}{lccccccc}
\hline & \# actuators & \# sensors & \multicolumn{2}{c}{ Actuators selected using vecnorm } & \multicolumn{2}{c}{ Actuators selected using MAC } \\
\cline { 5 - 6 } & & & Metric & Max force $[\mathrm{N}]$ & & Metric & Max force $[\mathrm{N}]$ \\
\hline Configuration 1 & 8 & 16 & 0.26 & 36 & 0.35 & 44 \\
Configuration 2 & 6 & 12 & 0.41 & 34 & 0.41 & 35 \\
\hline
\end{tabular}

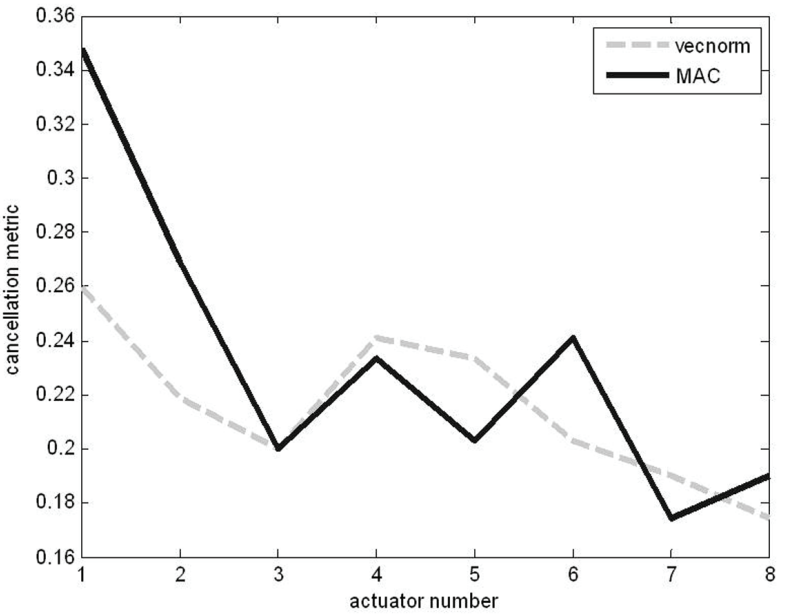

Fig. 4. Performance metric comparison of the top 8 actuator positions using the vector normalization and MAC approaches with error sensors in region 2 (main acoustic cavity).

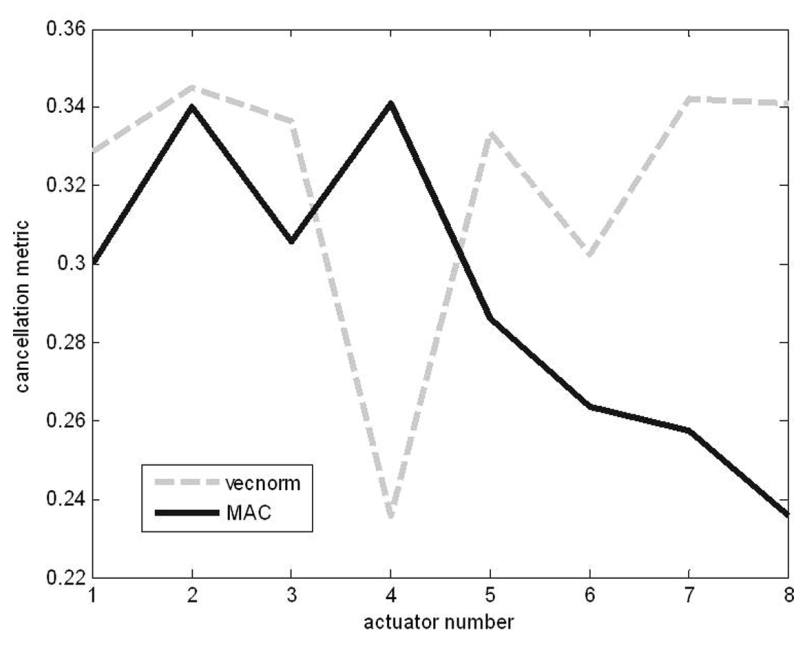

Fig. 5. Performance metric comparison of the top 8 actuator positions using the vector normalization and MAC approaches with error sensors in region 1 (sidewall cavity).

If there were no errors this metric should equal a vector of zeros at the sensor locations. In practice, cancellation is limited by the conditioning of the mathematical model. Since other limitations such as noise were not considered in this calculation, the cancellation achieved at the error sensors for a given location represents a best possible case. This metric was calculated for each actuator location to determine how well that location would perform, where a low value of the performance metric indicates a good position with more complete cancellation.

The computed metric for the top 8 actuator positions from all of the 6006 possible node locations on the skin of the barrel selected using the MAC and vector normalization approaches are shown in Fig. 4 for the lowest disturbance tone considered, $\mathrm{f} 1$, which is in the octave band centered at $125 \mathrm{~Hz}$. In this case, it was assumed that there were 16 error sensors distributed in region 2. The same metric was also evaluated using a random search of 500 of the nodal positions available. It is clear that both the locations using MAC and the vector normalization approach performed much better than the randomly selected locations with the means of the metric at $0.23,0.22$ and 0.65 for the MAC locations, the vector normalization locations and the random locations respectively. It is also clear from this exercise that there was not a significant difference between the MAC and the vector normalization approaches.

The results where the metric was calculated based on the same 16 sensor locations in region 2 but with error sensor locations selected in region 1 are shown in Fig. 5. The MAC and the vector normalization approach again out-performed the random locations with mean levels at $0.29,0.32$ and 0.56 for the MAC, the vector normalization, and the random locations respectively. The overall levels achieved with error sensors coincident with evaluation locations appear to be smaller showing that microphones placed in region 2 in a hardware implementation should give superior performance.

To compare candidate hardware configurations, it was assumed that the number of actuators would be half the number of error sensors. The assumption that there would be half as many actuators as sensors was based on practical considerations in the planned hardware demonstrations. No analytical justification of this assumption was undertaken, although the tools used could support such a study in future work. The means of the cancellation metric throughout region 2 determined for two different configurations are shown in Table 1 along with the maximum force required in each actuator set associated with the result. To derive a numerical value for force, it was necessary to 


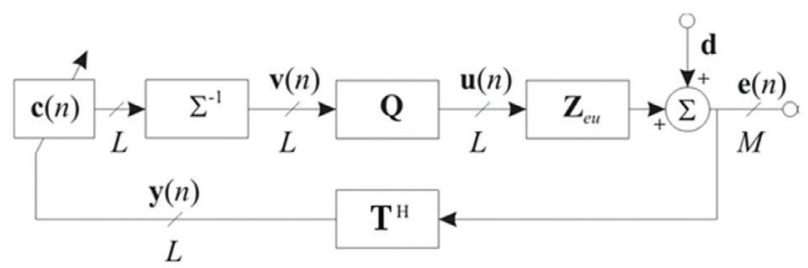

Fig. 6. Block diagram of principal component LMS algorithm in the complex frequency domain, with $\boldsymbol{L}$ actuators and $\boldsymbol{M}$ sensors.

assume a disturbance. An $80 \mathrm{dBA}$ maximum uncontrolled tone amplitude was chosen as this can be considered moderately loud for an interior space [3].

\section{Adaptive feedforward control approaches}

In practice, the controller $\mathbf{W}$ in Fig. 3 is often formed using an adaptive feedforward approach. The adaptation is necessary to compensate for small changes in the primary and secondary transfer function paths with time as well as changes of frequencies of disturbance inputs. By far the most pervasive adaptive feedforward algorithm is the filtered-x least mean square, FxLMS, algorithm [7]. In the FxLMS algorithm [15], the control filter coefficients are defined such that the error $\mathbf{E}(n)$ is minimized. They are given by

$$
\mathbf{W}(n+1)=\mathbf{W}(n)-\mu \mathbf{S}^{H} \mathbf{E}(n)
$$

where $\mu$ is the convergence coefficient, $\mathbf{S}^{H}$ is the Hermitian transpose of the matrix of filtered reference signals and $\mathbf{E}(n)$ is the error signal. Convergence speed is limited by the eigenvalue spread of $\mathbf{S}^{H} \mathbf{S}$.

An alternate approach [4] used to improve convergence is called the principal component LMS algorithm. In this case, the single frequency implementation of $\mathbf{G}_{e u}$ is renamed $\mathbf{Z}_{e u}$ and its SVD is defined as

$$
\mathbf{Z}_{e u}=\mathbf{T} \mathbf{\Sigma} \mathbf{Q}^{H}
$$

where $\mathbf{T}$ is the matrix of left singular vectors $\mathbf{Q}$ is the matrix of right singular vectors and $\mathbf{\Sigma}$ contains the singular values, $\sigma_{l}$, of $\mathbf{Z}_{e u}$. The SVD is used to transform the control problem as

$$
\mathbf{y}(n)=\mathbf{p}(n)+\sum \mathbf{v}(n)
$$

where

$$
\mathbf{y}(n)=\mathbf{T}^{H} \mathbf{e}(n), \mathbf{p}=\mathbf{T}^{H} \mathbf{d}(n) \text { and } \mathbf{v}(n)=\mathbf{Q}^{H} \mathbf{u}(n)
$$

with the resulting implementation shown in Fig. 6.

The adaptation is now implemented on $\mathbf{c}(n)$ where

$$
\mathbf{c}(n+1)=\mathbf{c}(n)-\alpha \mathbf{T}^{H} \mathbf{e}(n)
$$

where $\alpha$ is now the constant convergence coefficient. Similar to Eq. (7) for the FxLMS algorithm, convergence of the control signal is now limited by the eigenvalues spread of the matrix $\mathbf{T}^{H} \mathbf{T}$, which is equal to 1 since $\mathbf{T}$ is an all-pass matrix. This should result in an LMS algorithm with improved convergence properties.

The FxLMS approach was implemented in the analytical model to gain additional insight into Configurations 1 and 2 using both actuator location methods. The algorithm was allowed to run for 1000 seconds where the solution was changing less than $0.1 \%$ per second. This was not intended to represent the best possible performance achievable in an analytical study without noise but to provide a comparison to the optimal compensator approach for the same actuator positions and the similar levels of overall attenuation. Table 2 gives the analytical results for both algorithms and can be compared to Table 1 .

While the FxLMS results were not as dramatic in overall noise reduction, the maximum force requirements were significantly lower. Taking the optimal control and FxLMS results as a guide for hardware, between 6 and 8 actuators should have a force capability in the range of $8 \mathrm{~N}$ to $44 \mathrm{~N}$ at the f1 tone to expect global spatially averaged RMS cancellation of between 0.26 and 0.56 . In addition, the number of sensors necessary for a global reduction is between 12 and 16. 
Table 2

Mean of performance metrics for configurations 1 and 2 using FxLMS

\begin{tabular}{|c|c|c|c|c|c|c|}
\hline & \multirow[t]{2}{*}{ \# actuators } & \multirow[t]{2}{*}{ \# sensors } & \multicolumn{2}{|c|}{ Actuators selected using vecnorm } & \multicolumn{2}{|c|}{ Actuators selected using MAC } \\
\hline & & & Metric & Max force $[\mathrm{N}]$ & Metric & Max force $[N]$ \\
\hline Configuration 1 & 8 & 16 & 0.43 & 12 & 0.42 & 9 \\
\hline Configuration 2 & 6 & 12 & 0.46 & 15 & 0.56 & 8 \\
\hline
\end{tabular}

Table 3

Actuator comparison

\begin{tabular}{|c|c|c|c|c|c|}
\hline Name & Force constant [N/amp] & Coil resistance [Ohm] & Max power [W] & Total mass $[\mathrm{kg}]$ & Max force at $\mathrm{f} 1[\mathrm{~N}]$ \\
\hline Aura pro bass shaker & 6.6 & 4 & 75 & 1.4 & 29 \\
\hline CSA inertial actuator SA5 & 8.8 & Not given & Not given & 1.4 & 22 \\
\hline Motran IFX30-100 & 10.1 & 1.63 & 48 & 0.9 & 41 \\
\hline
\end{tabular}

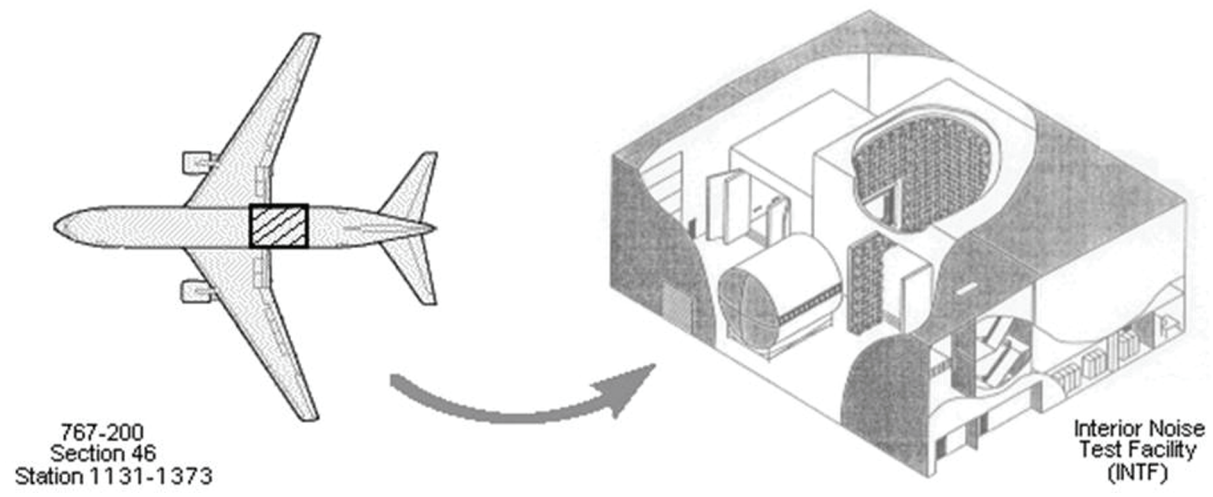

Fig. 7. Diagram of 767 barrel section and interior noise test facility (INTF).

\section{Testing}

The lab test involved a hardware demonstration of noise cancellation due to a tonal disturbance. The methods discussed in the analysis section were implemented on the hardware to define a customized modal test for actuator location. Several actuator configurations were tested for three separate tones to evaluate the potential for an active noise control system.

Testing was conducted in the Boeing Interior Noise Test Facility (INTF) in Seattle, WA. This facility consists of an anechoic chamber and a reverberation chamber. The chambers are both large enough to contain the test article, a $767-200$ barrel section. The barrel section is a fully furnished 767 that is $6.1 \mathrm{~m}$ long and $4.9 \mathrm{~m}$ diameter shown in Fig. 7.

The barrel section was cut from an irreparably damaged 767-200 and transported to Seattle for noise testing in the INTF facility. It was cut from Section 46 (fuselage portion behind the wing) which contains the cargo door. Both ends of the barrel are closed off with double layered acoustic walls. The acoustic walls are sufficiently high in transmission loss to ensure the cabin noise in the interior cabin comes through the fuselage sidewall and not through the ends as shown in Fig. 8.

The interior of the 767 barrel section has an interior from a 767-400. The interior includes everything that would be in a production passenger aircraft, including insulation, lining panels, stowage bins, seats and even air conditioning ducts as shown in Fig. 9. The INTF 767 barrel provides a very realistic simulation of an aircraft interior and provides a convenient tool for model validation [20].

The structural actuators selected were proof mass actuators due to their ease of integration and commercial availability. Three models of commercially available proof mass actuators were considered for the hardware implementation. The first four columns of Table 3 give the vendor-provided specifications of each of the actuators considered, and the fifth column gives the derived max force at $\mathrm{f} 1$ assuming that $\mathrm{f} 1$ is well above the resonance of the device. 


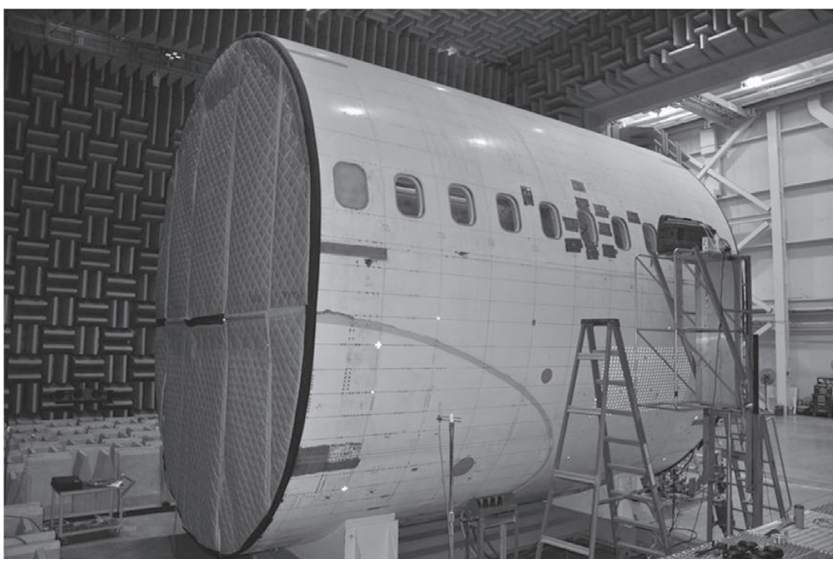

Fig. 8. 767-200 fuselage resting in cradles in INTF anechoic chamber.

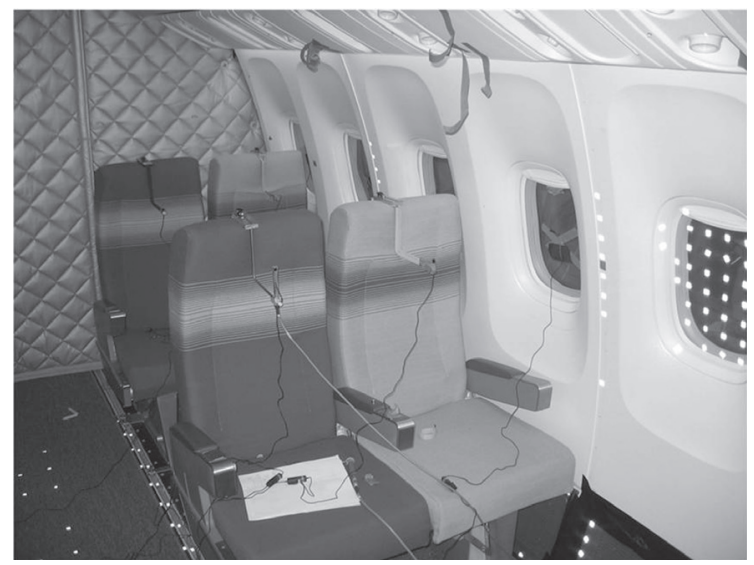

Fig. 9. Interior of 767 barrel section with passenger seats. Acoustic wall is visible in background.

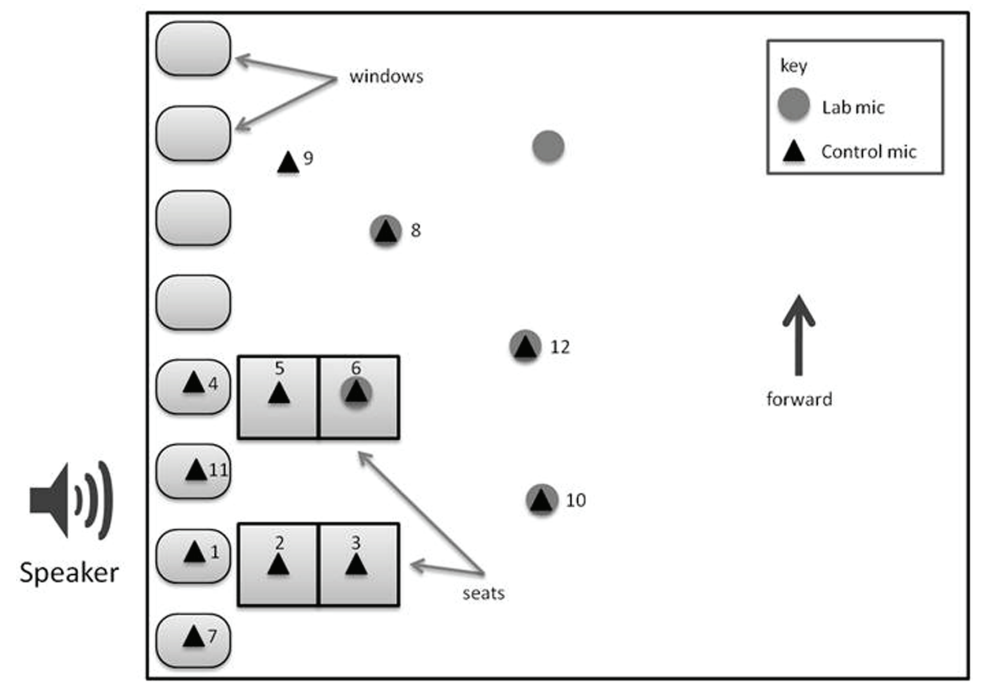

Fig. 10. Schematic of experiment.

In the case of the Aura and Motran products, this is derived by assuming that the maximum applied force simplifies to $k \sqrt{\frac{P}{R}}$ well above resonance where $k$ is the motor force constant, $P$ is max power and $R$ is resistance or the coil. Inductance of the coil is neglected in this calculation. For the case of the CSA product, the rated output at high frequency is provided.

Since all of the actuators gave maximum force numbers within the range identified by the analysis, the decision to select the Aura Pro Bass Shaker was governed by its availability and low cost due to significant penetration into the commercial audio market.

Sensor locations were intended to emphasize locations on seats that were at or near the head locations of passengers. As shown in Fig. 10, there were four control microphones at or near the head locations on each of the seats and four control microphones at or near the windows closest to the seats. The control microphones used were ATR3350 Lavalier microphones with microphone elements upgraded to provide higher sensitivity than the base model. High quality laboratory microphones (B\&K 4192) were also used to provide redundancy for the relatively inexpensive, consumer market control sensors and one independent measurement as shown in Fig. 10. One of the B\&K 4192 microphones was collocated with a control microphone at one of the seats.

The disturbance speaker was positioned $0.2 \mathrm{~m}$ from the barrel skin immediately outside the location where the 


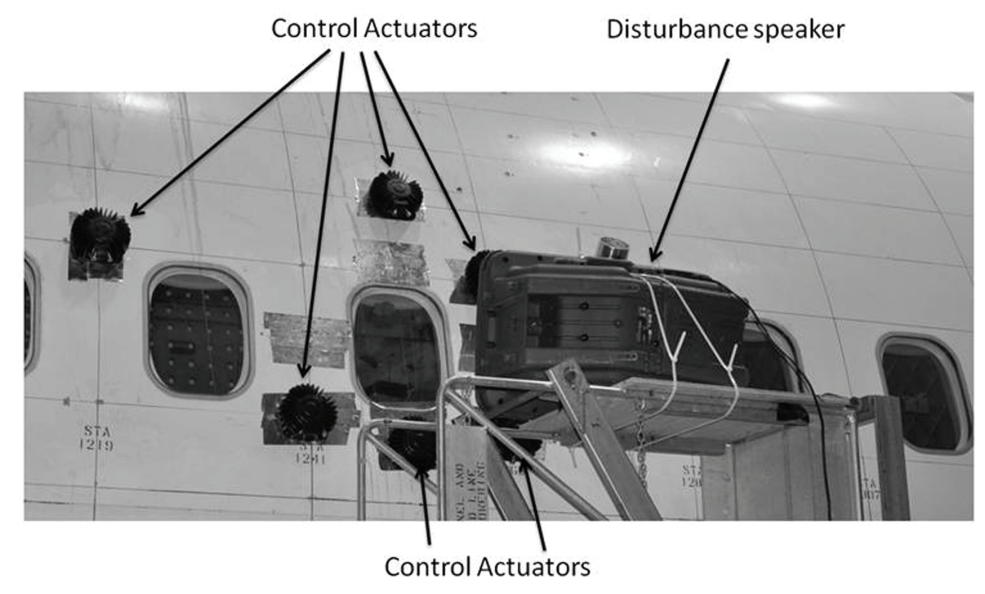

Fig. 11. Photograph of disturbance speaker and control actuators for $\mathrm{fl}$ tone.

seats were located as shown in Figs 8 and 11. The speaker was a JBL EON 15P-1 self powered system. The amplitude level selected at the speaker was adjusted so that the signal to noise levels on the interior microphones were satisfactory. A microphone located approximately $3 \mathrm{~m}$ from the disturbance speaker on the outside of the barrel measured $94 \mathrm{~dB}$ during the testing of the $\mathrm{f} 1$ tone. Assuming no room reflection, propagation where $r$ is the distance from the loudspeaker and pressure doubling at the skin surface, this means that the pressure directly outside the skin was $124 \mathrm{~dB}$. In the experiment, two higher tones were also included as disturbances, $\mathrm{f} 2$ and $\mathrm{f} 3$. These tones were located in the $1 / 3$ octave bands centered at $160 \mathrm{~Hz}$ and $250 \mathrm{~Hz}$ respectively. The same calculation for the f2 and $\mathrm{f} 3$ tones give $119 \mathrm{~dB}$ and $118 \mathrm{~dB}$ respectively.

Both the vecnorm and MAC ranking approaches to selecting actuator locations were programmed into dSPACE hardware with the DS1005 Power PC real time processor. The disturbance vector described in the analysis section was first measured between the voltage into the disturbance speaker and the signals from all of the control microphones. A PCB 086D20 force hammer with a $3 \mathrm{lb}$ head was then used to measure transfer functions between 20 candidate locations on the skin and all of the control microphones. Both metrics were automatically calculated at the frequency of interest and the best locations were selected. Figure 11 shows a photograph of 6 actuators attached to the skin at positions selected using the MAC method for the $\mathrm{f} 1$ tone. Although they were located on the outside of the aircraft for the testing, in practice they would be placed beneath the trim panel.

The ranking process was repeated several times to evaluate the utility of both the MAC and vecnorm metrics. In practice, the MAC metric consistently gave the same top actuator locations whereas the vecnorm metric did not. It was not conclusively determined why vecnorm failed to give consistent results, but it was suspected that this metric was more susceptible to error due to measurement noise. The effectiveness of the controller using these locations was not compared to that of using random locations as in the analytical work due to the difficulty associated with moving the actuators to new locations and the daunting number of possible locations.

\section{Adaptive noise cancellation results}

Both variants of the LMS algorithm were also programmed on the dSPACE hardware as Simulink native S functions. In addition, a shunt resistor was put in series with each of the actuators to monitor current to each actuator. Voltage to each actuator was monitored as well as the product of current and voltage during each control experiment. In this way, the power to the actuator was monitored. To avoid spillover, a bandpass filter centered on the frequency of the tone to be controlled was implemented on each microphone input to the controller. The principal component adaptation of the LMS algorithm was found to have slightly superior performance results on the hardware compared to the FxLMS approach. Figures 12 and 13 show the performance of both algorithms with an overall spatially averaged reduction of $10.3 \mathrm{~dB}$ for the FxLMS algorithm and $11.3 \mathrm{~dB}$ for the principal component LMS algorithm. 
Table 4

Overall results for all tones using both algorithms in anechoic room

\begin{tabular}{llccc}
\hline Tone & Algorithm & Reduction $[\mathrm{dB}]$ & Max power [W] & Mean power [W] \\
\hline f1 & FxLMS & 10 & 14 & 5 \\
& PC LMS & 11 & 50 & 20 \\
f2 & FxLMS & 4 & 31 & 12 \\
& PC LMS & 11 & 53 & 14 \\
f3 & FxLMS & 4 & 23 & 9 \\
& PC LMS & 8 & 36 & 23 \\
\hline
\end{tabular}

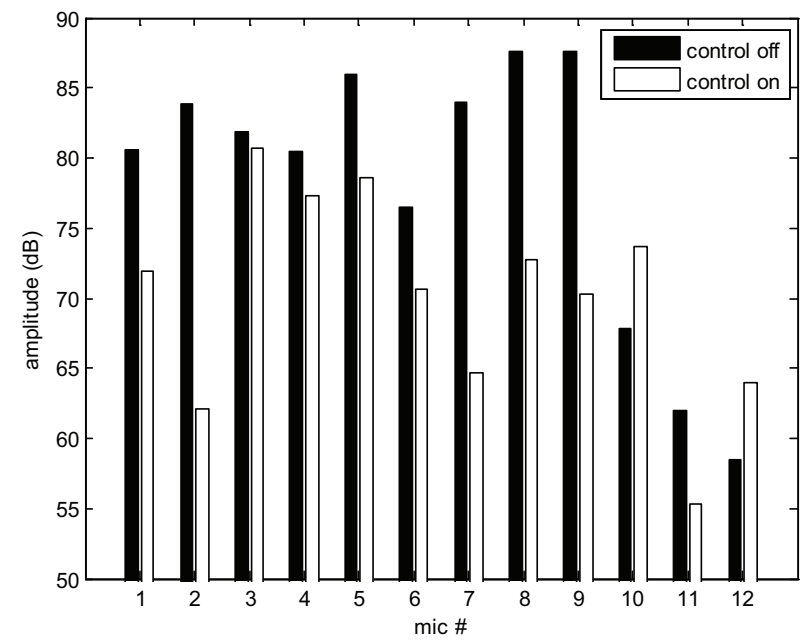

Fig. 12. FxLMS results for $\mathrm{f1}$ tone.

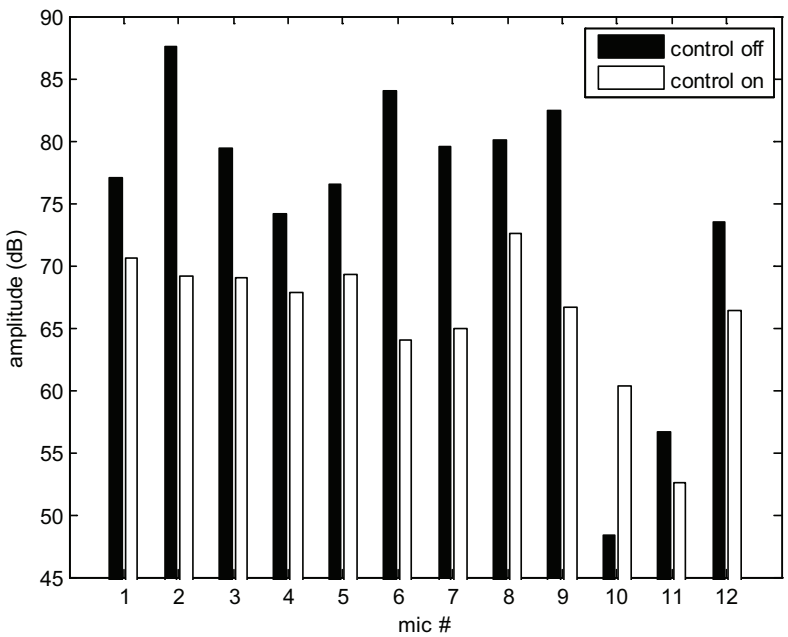

Fig. 14. Principal component LMS results for $\mathrm{f} 2$ tone.
Table 5

Overall results for all tones in reverb room

\begin{tabular}{lcccc}
\hline Tone & Algorithm & Reduction [dB] & Max power [W] & Mean power [W] \\
\hline f1 & PC LMS & 13 & 43 & 17 \\
f2 & PC LMS & 7 & 56 & 16 \\
f3 & PC LMS & 4 & 70 & NA \\
\hline
\end{tabular}

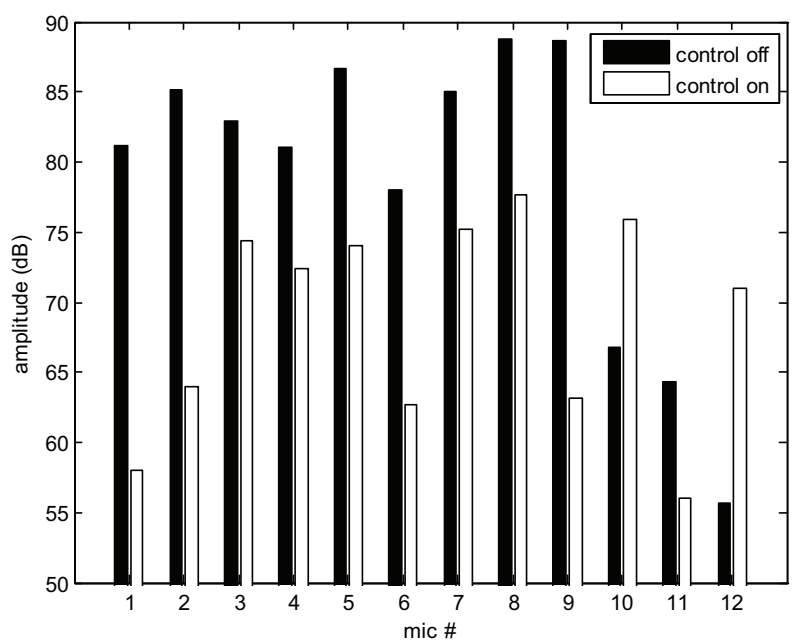

Fig. 13. Principal component LMS results for $\mathrm{f} 1$ tone.

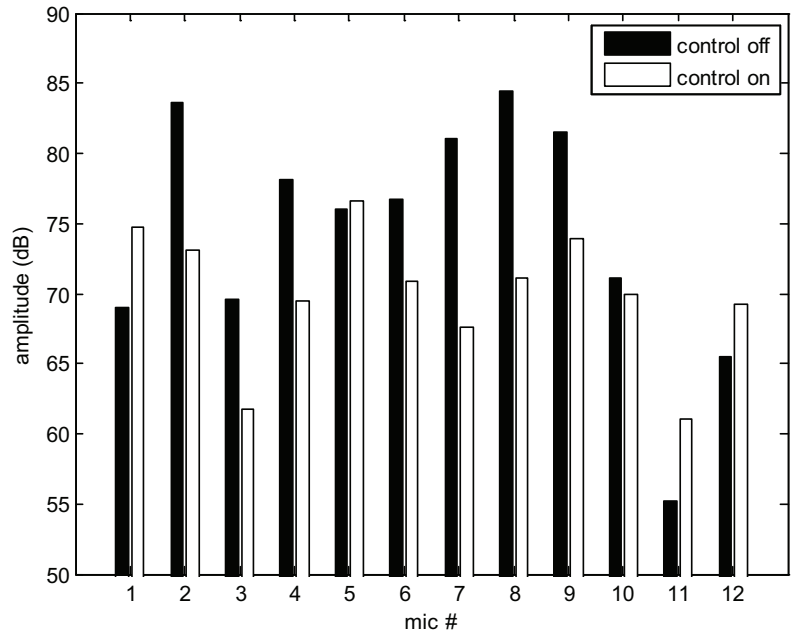

Fig. 15. Principal component LMS results for $\mathrm{f} 3$ tone.

Most of the microphone locations experienced reduced levels using both approaches with increases only at locations that were relatively low amplitude with the control off.

Both algorithms were also implemented at $\mathrm{f} 2$ and $\mathrm{f} 3$. The results for the principal component LMS algorithm at the higher frequency tones are shown in Figs 14 and 15. In every case, the overall level is reduced with the spatially averaged reductions shown in Table 4 along with the maximum power required of all 6 actuators and the RMS value. It was not determined why sound levels at some relatively "quiet" locations increased, but this, 
along with overall decreasing performance at higher frequency, suggests more actuators might lead to better overall performance. The principal component algorithm also consistently outperformed the FxLMS algorithm, but power levels were significantly higher.

Limited testing was also performed in the reverberation room for comparison with anechoic room testing. This testing was performed using only the principal components LMS algorithm. These results are shown in Table 5. The power result as calculated by instantaneous current times voltage for $\mathrm{f} 3$ exceeded the max power capability of the amplifier. This is indicative of a voltage command to the amplifier that was clipped. The actual max power set by the clipping limit of the amplifier of $70 \mathrm{~W}$ is recorded in the table. The reduced effectiveness at higher frequencies in the reverberation room was thought to be due to the many flanking paths of the disturbance. When the disturbance is more localized, as in the anechoic room, the role of the actuators is to disrupt the disturbance path. When the disturbance is distributed, as in the reverberation room, the role of the actuators is to make the skin behave like a speaker to cancel the sound at the error sensors. As the wavelength decreases in size, the cancellation of the distributed disturbance would be more effectively done with distributed speakers in the interior of the aircraft.

\section{Conclusion}

A system thus described is capable of significant low frequency noise reduction in a multi tonal acoustic disturbance environment representative of an open fan aircraft using adaptive noise cancellation and structural actuators. The use of a fully coupled structural acoustic model facilitated the design of a hardware demonstration including the number of sensors and actuators, locations and force capability of the structural actuators. The model also was used to derive a method to further refine actuator locations that leveraged modal testing techniques. Experimental results on an actual barrel fuselage section from a 767 aircraft showed that between $8 \mathrm{~dB}$ and $11 \mathrm{~dB}$ overall sound pressure reductions were achievable with individual reductions at most of the microphone error sensor positions. The PC LMS algorithm consistently outperformed the FxLMS algorithm in overall sound level reduction, but power levels required were significantly higher.

\section{Acknowledgments}

The authors wish to thank Dr. Dick Petersen of AECOM Australia Pty Ltd and Dr. Ben Cazzolato of University of Adelaide for providing adaptive feedforward tools and expertise.

\section{References}

[1] W.J. Albrecht, bnet. Retrieved 2010, from bnet Web site: findarticles.com/p/articles/mi_m0EIN/is_1997_April_16/ai_19322422, (16 April 1997).

[2] R. Allemang, The modal assurance criterion - twenty years of use and abuse, Sound and Vibration (2003), 14-21.

[3] L. Beranek, Noise and vibration control, Cambridge: INCE, 1971.

[4] R. Cabell, A principal component algorithm for feedforward active noise and vibration control, Blacksburg: Ph D Dissertation: Virginia Tech, 1998.

[5] M. Czech and A. Andersson, Fluidic wallpaper: An active control concept for low frequency broadband noise, 9th AIAA/CEAS Aeroacoustics Conference and Exhibit, Hilton Head, SC (2003).

[6] C. Dorling, G. Eatwell, S. Hutchins, C. Ross and S. Sutcliffe, A demonstration of active noise reduction in aircraft cabin, Journal of Sound and Vibration 128(2) (1989), 358-360.

[7] S. Elliott, Signal processing for active control, London: Academic Press, 2001.

[8] S. Elliott, P. Nelwon, I. Stothers and C. Boucher, In-flight experiments on the active control of propeller-induced cabin noise, Journal of Sound and Vibration 140(2) (1990), 219-238.

[9] C.R. Fuller and J.D. Jones, Experiments on reduction of propeller induced interior noise by active control of cylinder vibration, Journal of Sound and Vibration 112(2) (1987), 389-395.

[10] S. Griffin, S. Lane, C. Hansen and B. Cazzolato, Active structural acoustic control of a rocket fairing using proof-mass actuators, AIAA Journal of Spacecraft and Rockets 38(2) (2001), 219-225.

[11] S. Hirsch, N. Meyer, P. Westervelt, F. Li, M. Petrova and J. Sun, Experimental study of smart segmented trim panels for aircraft interior noise control, Journal of Sound and Vibration 231(4) (2000), 1023-1037. 
[12] S. Hirsch, J. Sun and M. Jolly, An analytical study of interior noise control using segmented panels, Journal of Sound and Vibration 231(4) (2000), 1007-1021.

[13] J. Juang and R. Pappa, An eigensystem realization algorithm for modal parameter identification and model reduction, Journal of Guidance and Control and Dynamics 8(5) (1984), 600-627.

[14] K. Kochan and D. Sachau, Robust active noise control in the loadmaster area of a military, Journal of the Acoustical Society of America 5 (May 2011), 3011-3019.

[15] S. Kuo and D. Morgan, Active noise control systems, John Wiley and Sons Inc, 1996.

[16] D. Li, L. Cheng and C. Gosselin, Optimal design of PZT actuators in active stuctural acoustic control of a cylindrical shell with a floor partition, Journal of Sound and Vibration 269(3-5) (2004), 569-588.

[17] K. Lyle and R. Silcox, A study of active trim panels for noise reduction in an aircraft fuselage, General Corporate and Regional Aviation Meeting and Exposition, Witchita, KS, 1995.

[18] D. MacMartin, Collocated structural control for reduction of aircraft cabin noise, Journal of Sound and Vibration 190(1) (1996), $105-119$.

[19] G. Mathur and B. Tran, Aircraft cabin noise reduction tests using active structural acoustic control, 15th AIAA Aeroacoustics Conference, Long Beach, CA (1993).

[20] J. Montgomery, Structural acoustic testing and FEM/BEM modelling of a 767 fuselage section, 6th AIAA/CEAS Aeroacoustics Conference and Exhibit (2000).

[21] S. Sharp, G. Koopman and W. Chen, Transmission loss characteristics of an active trim panel, NoiseCon (1997), 149-160.

[22] R. Silcox, C. Fuller and H. Lester, Mechanisms of active control in cylindrical fuselage structures, AIAA Journal 28(8) (1990), $1397-1404$.

[23] M. Simpson, T. Luong, C. Fuller and J. Jones, Full-scale demonstration tests of cabin noise reduction using active vibration control, Journal of Aircraft 28(3) (1991), 208-215.

[24] S. Snyder and C. Hansen, Using multiple regression to optimize active noise control system design, Journal of Sound and Vibration 148(3) (1991), 537-542.

[25] C. Testa, G. Bernardini and M. Gennaretti, Aircraft cabin tonal noise alleviation through fuselage skin embedded piezoelectric actuators, Journal of Vibration and Acoustics 133(051009) (October 2011), 1-10. 

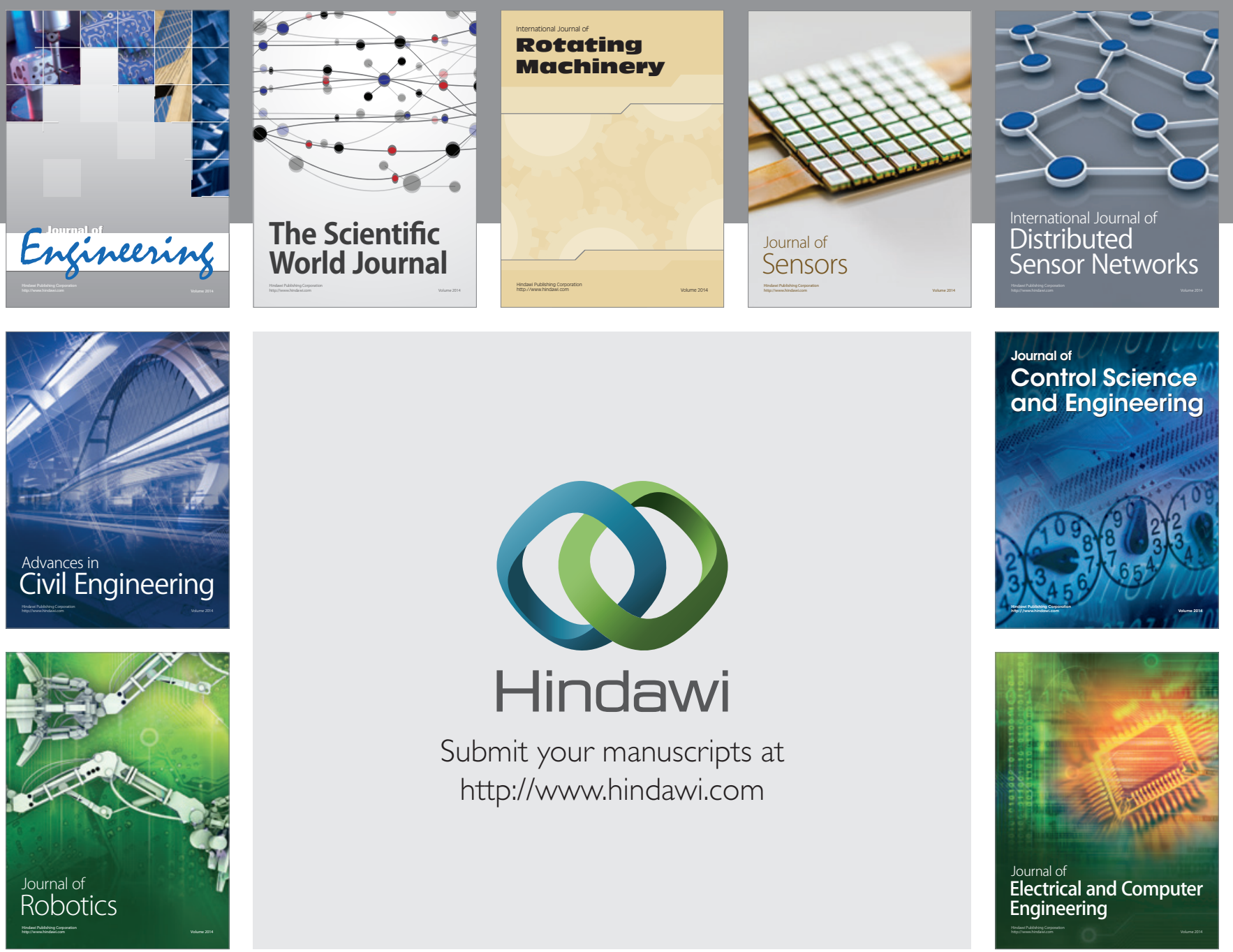

Submit your manuscripts at

http://www.hindawi.com
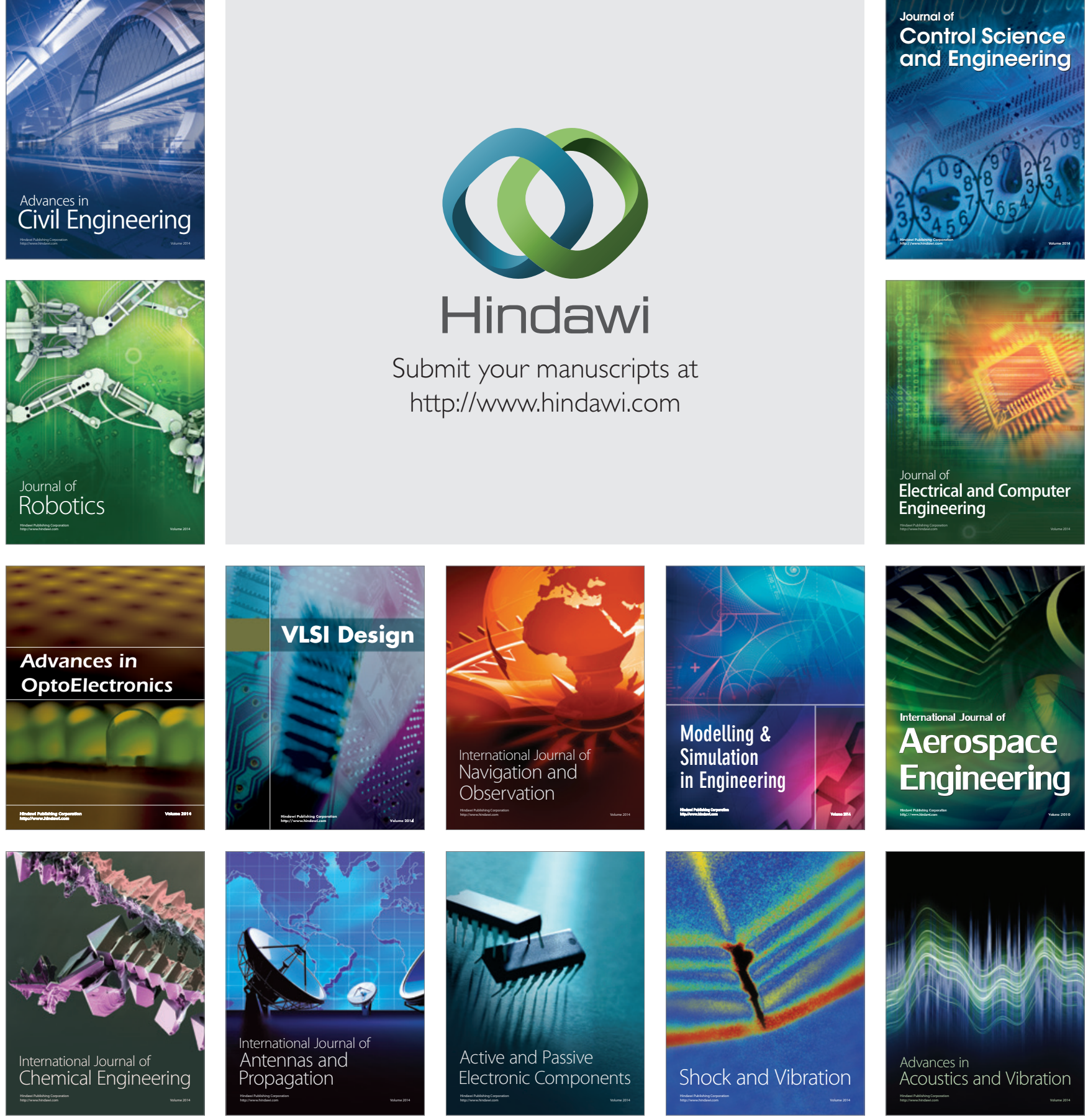